\title{
Pegaptanib sodium treatment in neovascular age-related macular degeneration: clinical experience in Germany
}

\author{
Nikolaus Feucht \\ Huebner Matthias \\ Chris P Lohmann \\ Mathias Maier \\ Augenklinik rechts der Isar, Technical \\ University Munich, Germany
}

Background: The VEGF Inhibition Study In Ocular Neovascularisation (VISION) reported the efficacy of intravitreal (ITV) vascular endothelial growth factor (VEGF) inhibition with pegaptanib sodium $\left(\right.$ Macugen $^{\circledR}$ ) for the treatment of neovascular age-related macular degeneration (AMD). This paper reports clinical experience with pegaptanib sodium for the treatment of occult or minimally classic choroidal neovascularization (CNV) due to AMD.

Material and methods: The study included 50 eyes (in 49 patients) with either occult CNV or minimally classic CNV secondary to neovascular AMD who were not eligible for photodynamic therapy (PDT). Study data were analyzed retrospectively. During the 6-month study, patients were administered an average 2.74 injections of $0.3 \mathrm{mg}$ ITV pegaptanib sodium. Angiography and optical coherence tomography (OCT) examinations were carried out and intraocular pressure (IOP) and visual acuity (VA) were measured at baseline, at 3 months and at 6 months. An eye examination was performed and VA was measured the 2 days following treatment and then again at weeks $4-6$, and at 3 and 6 months. OCT, VA, and IOP were also assessed at 1 month.

Results: ITV pegaptanib sodium was well tolerated and no treatment complications arose. Mean VA was measured as: $0.37 \pm 0.24$ at baseline; $0.37 \pm 0.25$ at 1 month; $0.37 \pm 0.25$ at 3 months and $0.40 \pm 0.26$ at 6 months. VA was stabilized in approximately $90 \%$ of eyes treated with pegaptanib sodium. OCT examination showed a minimal change in central retinal thickness (CRT) during the course of the study, from $251.19 \mu \mathrm{m}$ at baseline to $251.63 \mu \mathrm{m}$ at 6 months. No elevation in IOP was measured during treatment at 4-6 months in patients receiving pegaptanib sodium.

Conclusions: ITV therapy with pegaptanib sodium for occult and minimally classic CNV secondary to neovascular AMD offered good efficacy with a favorable adverse events profile. The majority of patients showed stabilization in all assessed parameters. In clinical practice, careful consideration should be given to the use of nonselective VEGF inhibition in patients with a high cardiovascular risk profile or in those with a history of thromboembolic events.

Keywords: Intravitreal (ITV) injection, age-related macular degeneration (AMD), choroidal neovascularization (CNV), anti-VEGF therapy, pegaptanib sodium

\section{Introduction}

Age-related macular degeneration (AMD) is one of the most common causes of irreversible, severe vision loss in the elderly population of Western societies, often having a significant effect on quality of life (Bird 1996; Mitchell and Bradley 2006).

Neovascular AMD is characterized by choroidal neovascularization (CNV). The pathophysiology of neovascular AMD is complex and involves numerous processes. Vascular endothelial growth factor (VEGF) plays a key role in many of these processes 
(Adamis and Shima 2005). VEGF is a stimulator of the angiogenesis associated with CNV: it plays a fundamental role in the stimulation of cell proliferation of the endothelium, increases vascular permeability and has a chemotactic effect on macrophages. Yet VEGF also induces blood vessel growth and is necessary for the preservation of normal vessels and vascular function (Thakker et al 1999; Tilton et al 1999; Bernatchez et al 2002).

Pegaptanib sodium (Macugen ${ }^{\circledR}$, [OSI] Eyetech Pharmaceuticals, Pfizer) was the first anti-VEGF agent licensed for the treatment of neovascular AMD. It is an oligonucleotide that functions as an aptamer (from the Latin "aptus", to match/fit; and the Greek "meros", part) and, similar to an antibody, specifically blocks the activity of the $\mathrm{VEGF}_{165}$ isoform that is largely responsible for the formation of the CNV associated with neovascular AMD (Ishida et al 2003).

The efficacy of pegaptanib sodium was investigated in the VEGF Inhibition Study In Ocular Neovascularization (VISION): 2 concurrent prospective, randomized, doubleblind, multicenter, dose-ranging, controlled clinical trials (Gragoudas et al 2004). VISION defined broad inclusion parameters, including patients with all angiographic subtypes (predominant classic, minimally classic and occult lesions) and with visual acuity (VA) between 20/320 and 20/40. Patients were randomized to receive intravitreal (ITV) injections of pegaptanib sodium $(0.3 \mathrm{mg}, 1.0 \mathrm{mg}$, or $3.0 \mathrm{mg}$ ) or sham injection in 1 eye every 6 weeks over a period of 48 weeks. The study included all patients with classic and occult $\mathrm{CNV}$, despite the fact that patients with predominantly classic lesions could have received additional photodynamic therapy (PDT) if it was considered appropriate by the investigator. The primary endpoint was the proportion of patients who had lost fewer than 15 letters ( 3 lines) of VA at 54 weeks and at years 2 and 3 (VA stabilization was defined as a loss of fewer than 15 letters over the duration of the trial).

VA stabilization was achieved in approximately $70 \%$ of patients administered pegaptanib sodium compared with $55 \%$ in the control group ( $p<0.001)$. VISION concluded that pegaptanib sodium was effective in all subtypes of neovascular AMD, including predominant classic, minimally classic, and occult lesion types. The treatment benefit was not only noted after 54 weeks, but also at all examinations carried out during years 1 and 2 of the study (Gragoudas et al 2004; D’Amico et al 2006). During the second year, the therapeutic effect was maintained through administration of 6-weekly ITV injections (D’Amico et al 2006).
In this review, we report the findings of our clinical experience with the agent over a period of 6 months at our clinic in Germany.

\section{Material and methods}

The data reported were attained through clinical experience with compassionate use pegaptanib sodium in 50 eyes (of 49 patients) with progressive vision loss due to neovascular AMD, before and after the agent's approval in the European Union (EU).

At assessment, carried out up to a month prior to treatment initiation, patients were deemed eligible for treatment with pegaptanib sodium if they reported acute visual impairment or metamorphopsia and demonstrated occult or minimally classic CNV. Of the 50 eyes treated with pegaptanib sodium, 39 had occult CNV and 11 had minimally classic CNV. Most patients were female: 32 women compared with only 17 men, and had an average age of 78 years (age range of 54-87 years). Twenty-two of the patients had received previous treatment during the 3- to 6-month period prior to the first study injection. Of those 22 patients: 8 patients had received triamcinolone monotherapy; 1 had been treated with PDT monotherpay; 8 with PDT in combination with triamcinolone; 2 patients had received ITV injections of tissue plasminogen activator r-TPA and gas, and 3 had undergone cataract operations.

Baseline assessments included best-corrected VA (BCVA), slit lamp examination of the anterior segment, funduscopy, OCT, and measurement of IOP. The examinations were repeated at 4-6 weeks, at 3 months and at 6 months after the first injection. IOP and slit lamp examinations were also performed on days 1 and 2 post-injection. Fluorescein angiography (FA) of the ocular fundus was performed at baseline, post injection, at 3 months, and at 6 months. Baseline CRT and volume were assessed with OCT and compared with measurements taken at 1 month, at 3 months, and at 6 months post injection. All patients were monitored for a period of 6 months.

All patients received an ITV injection of pegaptanib sodium $(0.3 \mathrm{mg})$. Indicators of the need for re-injection were increased intra-retinal fluid or a rise in CRT of more than $100 \mu \mathrm{m}$ (measured by OCT), a decrease in VA or angiographically persisting leakage. An average of 2.74 injections were administered per patient; the majority of patients received 1 (15 patients) or 2 (17 patients) injections, but 8 patients received 3 injections, 5 received 4 injections, and 5 patients received 5 injections. The high percentage of patients that received only 1 or 2 injections during the 6-month follow up 


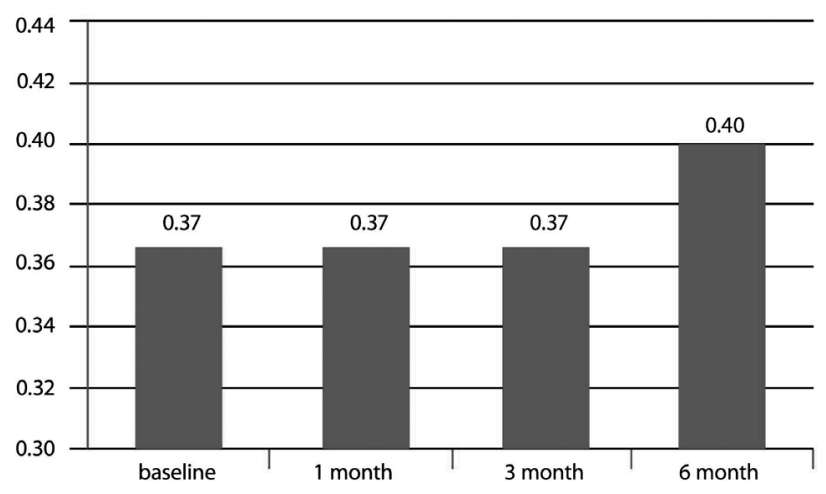

Figure I Mean visual acuity over time $(n=50)$.

period of this study was a consequence of the need to apply for reimbursement for each separate injection at the outset of the study. As a result, a standardized injection regimen could not be established in some cases. Patients were deemed ineligible for treatment if they had maculopathy originating from a condition other than AMD, or if they exhibited signs of any acute ocular infection.

The ITV injection of pegaptanib sodium $(0.3 \mathrm{mg})$ was performed in accordance with the guidelines for ITV injection published by the Macula Committee of the German Ophthalological Society (DOG) and the Professional Association of Ophthalmologists of Germany (BVA) (DOG and BVA 2006). Before injection administration, the eye was washed with povidone iodide (5\%) and the ciliae and lid regions were then wiped, also with povidone iodine (5\%). The drape (as used for intraocular intervention) was applied, a speculum was inserted, and paracentesis was performed in all cases to reduce the intraocular volume. After dislocation of the conjunctivae (with the use of a sterile pad to avoid penetration of the conjunctiva and sclera at the same location), pegaptanib sodium $(0.09 \mathrm{~mL})$ was injected at a distance of $3.5 \mathrm{~mm}$ from the limbus. Antibiotic eye drops were then given.

\section{Results}

The 6-month results from the 50 treated eyes were analyze retrospectively. Mean VA at baseline was $0.37 \pm 0.24$ and remained stable at 1 month and 3 months $(0.37 \pm 0.25)$. At 6 months, however, the mean BCVA had improved to $0.40 \pm$ 0.26 (see Figure 1).

VA measurements at 1 month post injection revealed that: 2 patients (4\%) had lost 3 or more lines; 46 patients (92\%) had stabilized (had lost or gained less than 3 lines); and 2 patients (4\%) had gained more than 3 lines (see Figure 2a). VA measurements 3 months post injection demonstrated that:
1 patient (2\%) had lost 3 or more lines; 48 patients $(92 \%)$ had stabilized \pm 3 lines; and 1 patient (4\%) had gained more than 3 lines (see Figure 2b). At final measurement, 6 months post injection: only 1 patient (2\%) had lost more than 3 lines; 45 patients $(90 \%)$ had stabilized within \pm 3 lines; and 4 patients $(8 \%)$ had experienced an increase in VA of more than 3 lines (see Figure 2c).

Mean CRT, measured by OCT examination, at baseline was $251.19 \pm 75.83 \mu \mathrm{m}$. Post-injection measurements or CRT recorded mean values of: $252.00 \pm 71.27 \mu \mathrm{m}$ at 1 month; $247.38 \pm 66.28 \mu \mathrm{m}$ at 3 months; and $251.63 \pm 68.23 \mu \mathrm{m}$ at 6 months (see Figure 3).

Semi-quantitative evaluation of the angiographic images revealed stabilization or reduction in lesion size or exudation. During the 6-month period following first ITV administration of pegaptanib sodium, no conversion of occult $\mathrm{CNV}$ into a predominantly classic $\mathrm{CNV}$ was observed. One patient had an IOP of more than $21 \mathrm{mmHg}$ when measured 1 month post-injection, but this returned to normal after management with topical anti-glaucoma therapy. No serious post-injection complications (eg, intraocular bleeding, retinal detachment, endophthalmitis, or cataract progression) were observed.

The following case study offers an illustration of the approach to treatment taken in the study.

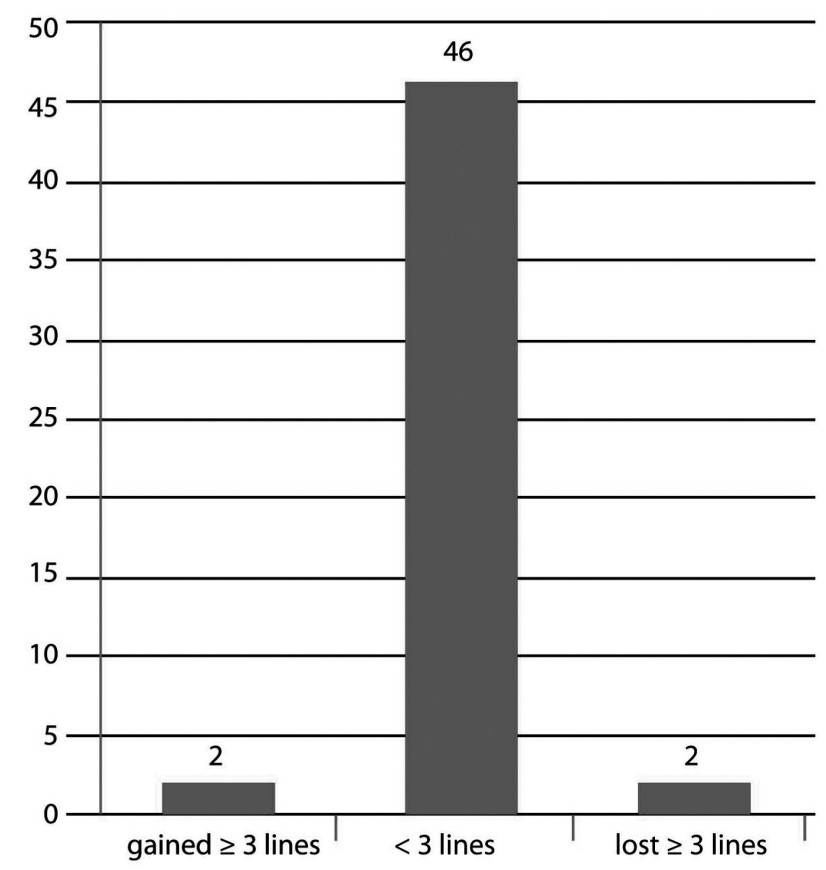

Figure $2 \mathrm{a}$ Change in visual acuity after I month $(\mathrm{n}=50)$. 


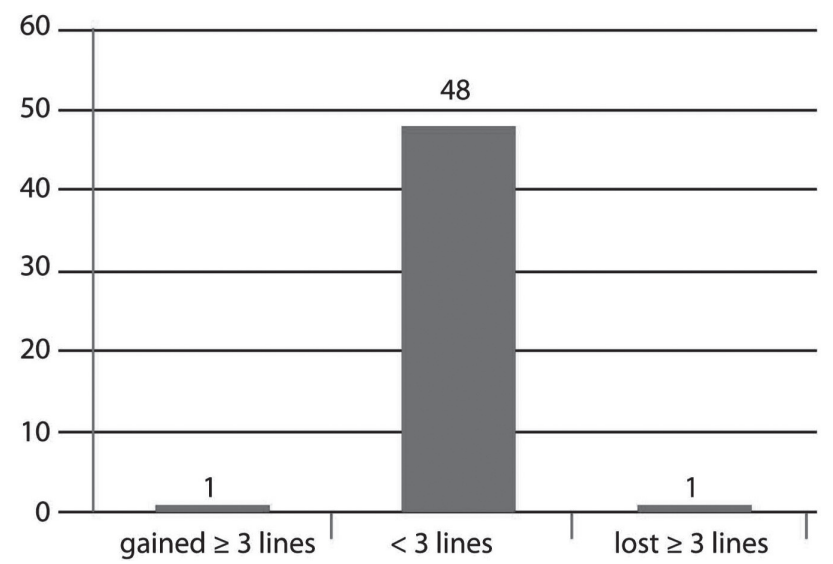

Figure $\mathbf{2 b}$ Change in visual acuity after 3 months $(n=50)$.

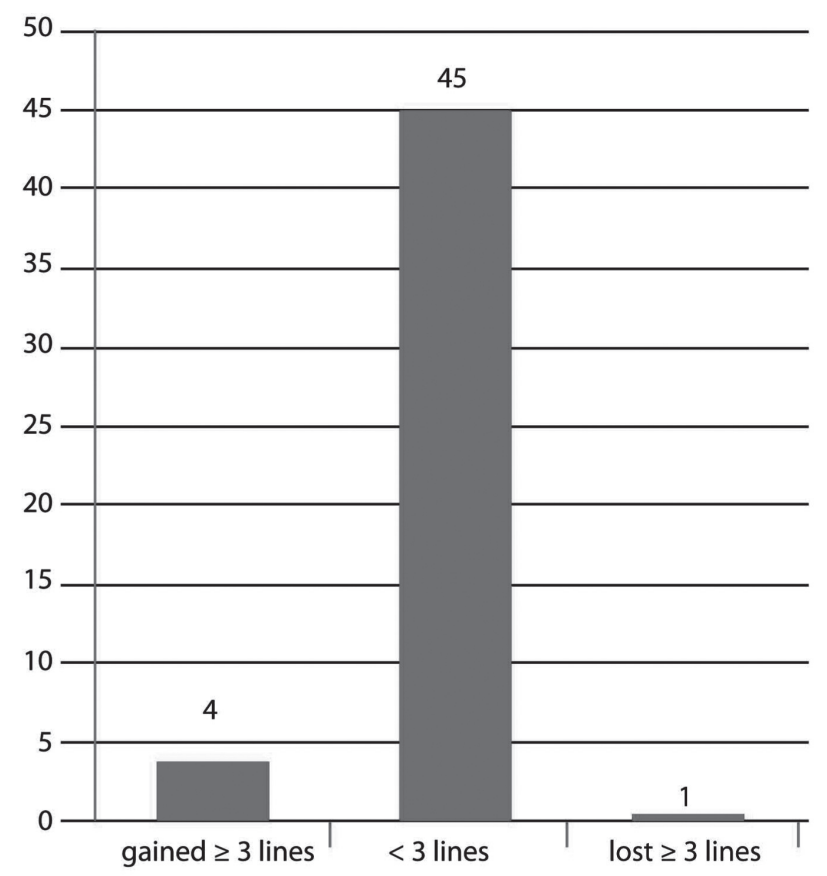

Figure 2c Change in visual acuity after 6 months $(n=50)$.

\section{Case study}

At baseline, Patient A complained of visual impairment and metamorphopsia in their better (left) eye. The right eye had a VA of approximately 0.03 and featured macular scarring due to neovascular AMD, and the left-eye had VA of 0.20 (see Figure 4). VA measured after the first ITV injection of pegaptanib sodium was 0.40 , which increased to 0.63 after 3 injections and stabilized at 0.60 after 5 injections. Figure 5 shows the angiographic findings after 5 injections with pegaptanib sodium $(0.3 \mathrm{mg})$. After only the second injection Patient A reported that they were able to read and drive again.

\section{Conclusion}

This paper is a report of our clinical experience with pegaptanib sodium for the treatment of neovascular AMD. As there were no approved therapies available for the treatment of occult and minimally classic CNV in 2005, patients who were administered pegaptanib sodium for treatment of neovascular AMD in September 2005 ahead of its EU licence did so through the compassionate use program (DOG and BVA 2006). Eleven patients at our clinic were treated with pegaptanib sodium in advance of its licence being granted for the treatment of neovascular AMD in Germany in May 2006; 8 of those patients received more than 1 injection of the agent.

A total of 35 eyes were treated with multiple injections in the observation period. There was good clinical acceptance of the drug with no observation of severe side effects, such as endophthalmitis or retinal detachment. Re-treatment was also well tolerated among the patient group. One patient developed an elevation in their IOP after the first injection, but this was quickly and successfully managed with the use of topical anti-glaucoma medication. In total, patients were observed for a period of 6 months and some $(4 \%-8 \%)$ benefited from an improvement in their vision of more than 3 lines. In comparison to the VISION trial there was a higher percentage of patients with stabilization of VA at the endpoint of our investigation (at month 6).

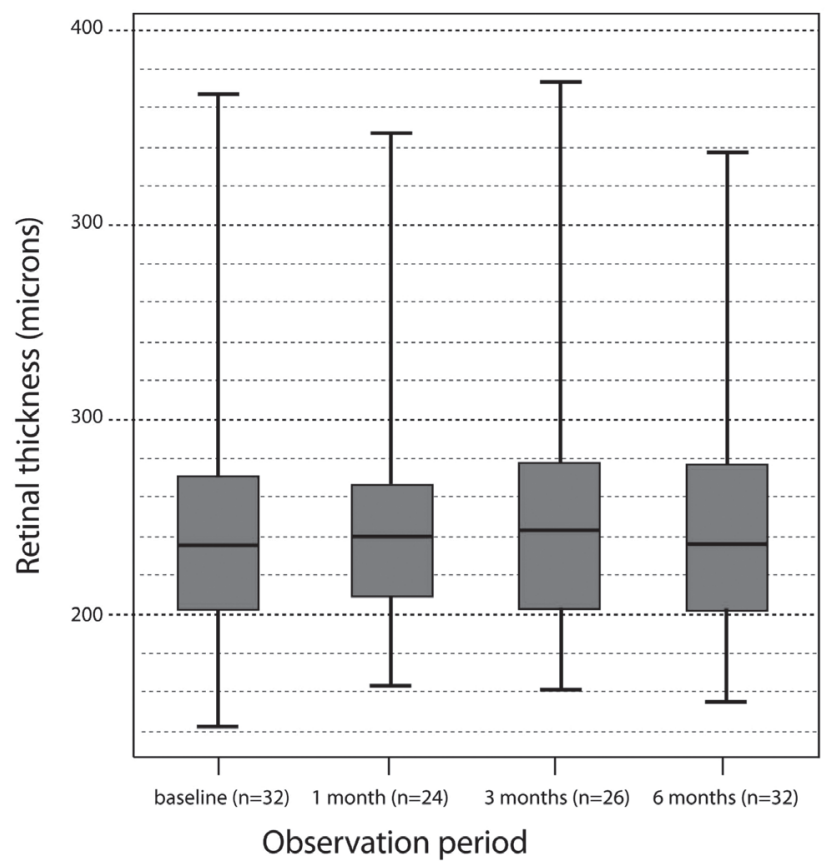

Figure 3 Central retinal thickness as measured with optical coherence tomography over time. 
Right eye: $\mathrm{VA}=0.03$
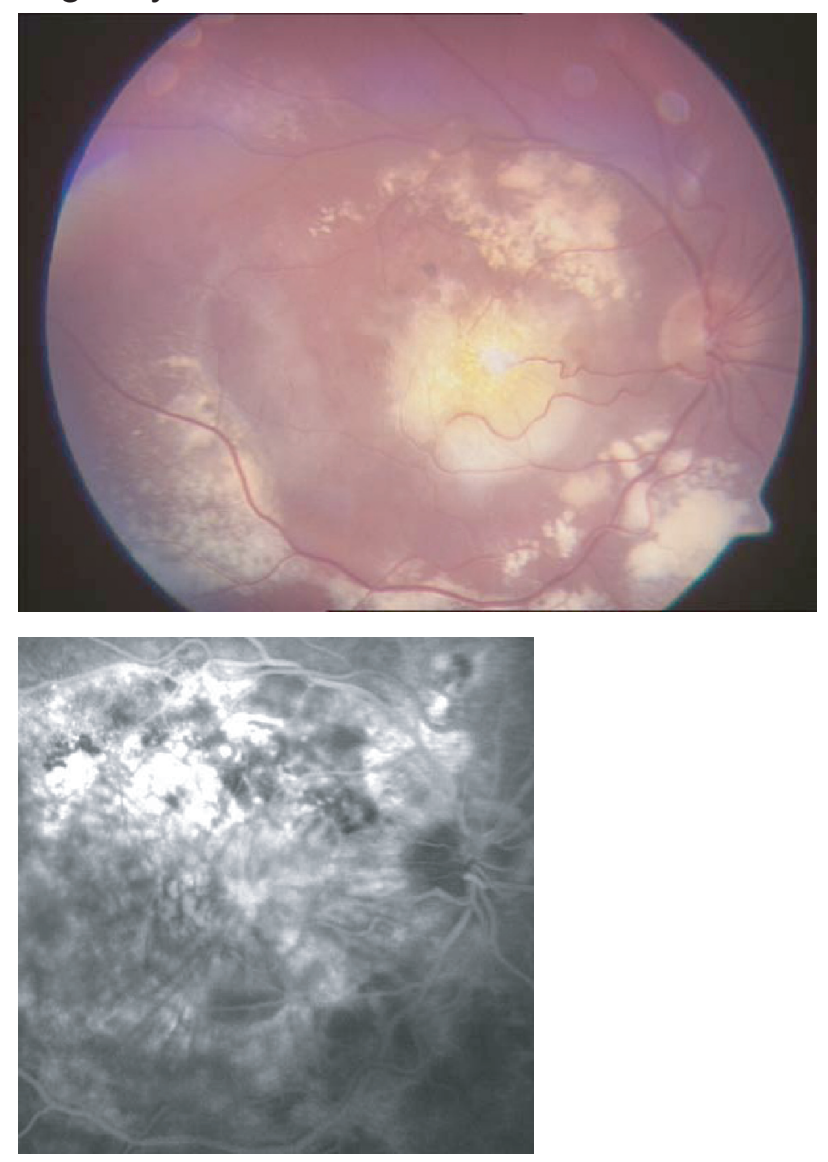

Left eye: $\mathrm{VA}=0.20$
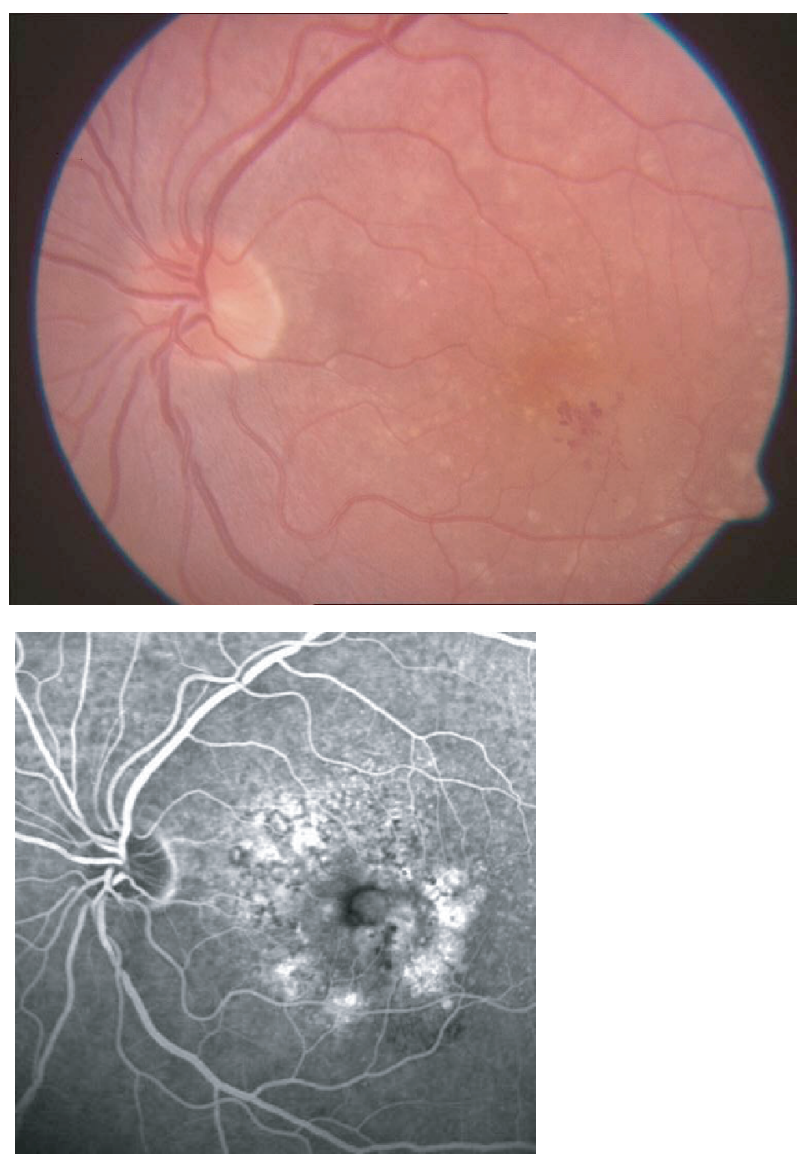

Figure 4 Patient A: aged 75 years, results of funduscopy and late-phase angiography assessment at baseline. Acute left-eye visual impairment and metamorphopsia.

No sub-analysis was performed to determine any possible relationship between lesion size and visual outcome. The OCT assessment of CRT at 1, 3, and 6 months post injection showed little change (see Figure 3). No correlation was observed between CRT and VA.

During the period of observation, 5 patients switched therapy from selective anti-VEGF therapy with pegaptanib sodium to treatment with unselective anti-VEGF agents because of a lack of efficacy. No further visual impairment was observed in these patients. The efficacy of ranibizumab (Lucentis ${ }^{\circledR}$, Genentech, Inc) for the treatment of neovascular AMD has been investigated in prospective, randomized, placebo-controlled multi-center studies: Minimally Classic/Occult Trial of the Anti-VEGF Antibody Ranibizumab in the Treatment of Neovascular Age-Related Macular Degeneration (MARINA), and Anti-VEGF Antibody for the Treatment of Predominantly Classic Choroidal Neovascularization in Age-Related Macular Degeneration (ANCHOR). The data showed that BCVA stabilization was achieved in approximately $90 \%$ of patients (stabilization was defined as a loss of less than 3 lines on the Early Treatment Diabetic Retinopathy Study [ETDRS]) chart (Brown et al 2006; Rosenfeld et al 2006a, b). No such large-scale randomized clinical trials have been carried out to assess the efficacy of bevacizumab (Avastin ${ }^{\circledR}$, Genentech, Inc) for ITV administration for neovascular AMD and the agent remains unlicensed for this indication at this time. However, the literature does report the findings of retrospective case control studies of up to 266 patients in which a decrease of CRT and an improvement of VA was reported in up to $38 \%$ of patients following treatment with ITV bevacizumab for neovascular AMD (Avery et al 2006; Spaide et al 2006).

ITV anti-VEGF therapy requires periodic injections and, consequently, re-treatment tolerability is important. Our experience of pegaptanib sodium indicates that repetitive treatment is well tolerated by patients. At the time of writing, there are currently no consistent recommendations to advise on the exact pre-treatment diagnostic measures, follow-up examinations (eg, single OCT vs OCT plus 

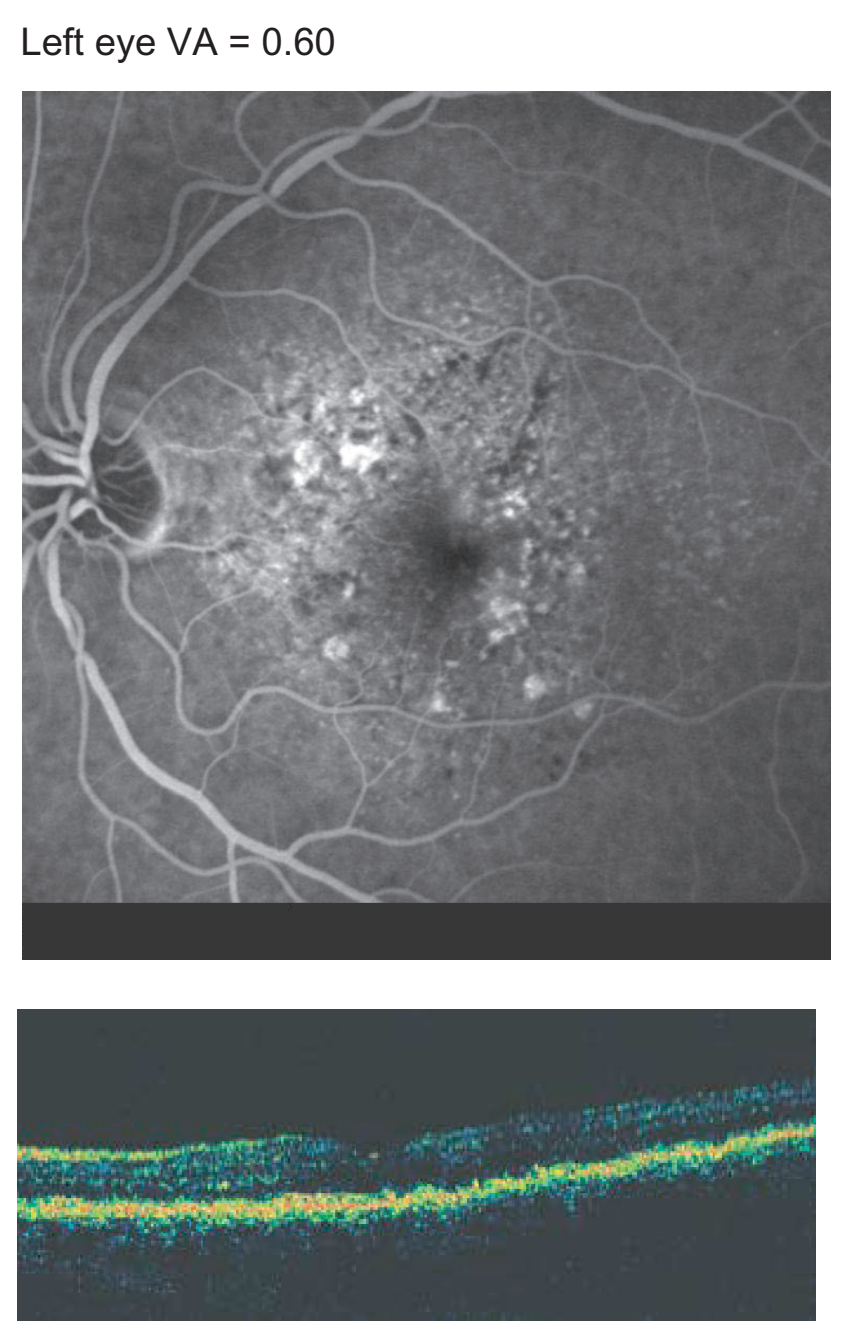

Figure 5 Patient A: results of left-eye optical coherence tomography and late-phase angiography after 5 injections of pegaptanib sodium.

angiography), or on the duration and frequency of follow-up assessments. In addition, it still remains unclear whether it is necessary to administer repeat injections every 6 weeks after initiation of a therapy for a 2 -year period, or if clinical judgement can be used to determine whether the interval between successive injections can be prolonged. Two recent trials investigated a loading strategy for ranibizumab therapy - the Prospective Optical Coherence Tomography (OCT) Imaging of Patients with Neovascular Age-Related Macular Degeneration (AMD) Treated with Intra-Ocular ranibizumab (PrONTO), and a Phase IIIb, multicenter, randomized, double-masked, sham Injection-controlled study of the Efficacy and safety of Ranibizumab in subjects with subfoveal CNV with or without classic CNV secondary to AMD (PIER). The approach involved the administration of 3 injections with subsequent re-treatment being administered as needed. The strategy appeared to result in superior treatment outcomes than those achieved when there are prolonged intervals between the re-treatment injections (Rosenfeld et al 2006c).

Pegaptanib sodium was the first anti-VEGF agent approved for ITV administration for neovascular AMD. The VA results of the VISION study are clearly inferior to those of the MARINA and ANCHOR studies with ranibizumab. At the time of writing, therefore, pegaptanib sodium is considered second-line therapy. However, VA efficacy is only one of the clinical considerations that must be taken into account. In our study, the safety profile of pegaptanib sodium during the observation period was good; no relevant systemic or ocular side effects were noted. Cardiovascular incidents and overall mortality in the pegaptanib sodium group were comparable to those of the sham injection group. In addition, a separate trial looking at ITV pegaptanib sodium injections over a 2-year period concluded that there was no clinical or angiographical-proven retinal or choroidal damage detectable (Gragoudas et al 2004). It should also be noted that there are currently no data concerning the safety of bevacizumab in the ocular environment. Systemic administration of the agent, as licensed for the treatment of colorectal cancer, has been noted to cause an elevation in the incidence of thrombembolic events, as well as gastrointestinal perforations (Roche Products Limited 2007).

The Safety Assessment of Intravitreal Lucentis fOR AMD (SAILOR) study investigated the safety profile of different dosages of ITV ranibizumab. After a follow-up period of 230 days, an elevated incidence of (cerebral) strokes was found in the study arm randomized to receive $0.5 \mathrm{mg}$ ITV ranibizumab compared with those randomized to $0.3 \mathrm{mg}$ ranibizumab. Patients with a history of apoplexy seemed to have a higher risk of apoplectic stroke. No difference was found for myocardial infarction or overall mortality (Dafer et al 2007). These data are of particular interest when considered in conjunction with animal studies that found ITV administration resulted in the substance being detectable systemically, indicating systemic bioavailability and a need to assume the possibility of associated side effects (Drolet et al 2000). This argument is further substantiated by reports in the literature of incidents where therapeutic effects were observed not only in the treatment eye, but also in the fellow eye (Mennel et al 2007).

The approval studies for pegaptanib sodium and ranibizumab clearly show that multiple injections are necessary over a prolonged period of time to maintain the effectiveness of therapy. It is also noteworthy that the implications of a permanent ocular or systemic blockade of all VEGF-A 
isoforms remain unclear at this time. In a clinical setting, therefore, it is critical to consider the potential systemic side effects of repeated treatments with nonselective VEGF inhibitors in those patients with a high cardiovascular risk profile or with a history of thromboembolic events.

\section{References}

Adamis AP, Shima TS. 2005. The role of vascular endothelial growth factor in ocular disease. Retina, 25:111-18.

Avery RL, Pearlman J, Pieramici DJ, et al. 2006. Intravitreal bevacizumab (Avastin) in the treatment of proliferative diabetic retinopathy. Ophthalmology, 113:1695.e1-15.

Bernatchez PN, Rollin S, Soker S, et al. 2002. Relative effects of VEGF$\mathrm{A}$ and VEGF-C on endothelial cell proliferation, migration and PAF synthesis: Role of neuropilin-1. J Cell Biochem, 85:629-39.

Bird AC. 1996. Age-related macular disease. Br J Ophthalmol, 80:1-2.

Brown DM, Kaiser PK, Michels M, et al; ANCHOR Study Group. 2006. Ranibizumab versus verteporfin for neovascular age-related macular degeneration. $N$ Engl J Med, 355:1432-44.

Dafer RM, Schneck M, Friberg TR, Jay WM. 2007. Intravitreal ranibizumab and bevacizumab: a review of risk. Semin Ophthalmol, 22:201-4.

D'Amico DJ. 2005. Results of the second year of Macugen for the treatment of neovascular AMD (V.I.S.I.O.N). Abstract presented at the American Society of Retinal Specialists Meeting, Montreal, 16-20 July 2005.

D'Amico DJ, Masonson HN, Patel M, et al. 2006. Pegaptanib sodium for neovascular age-related macular degeneration: two-year safety results of the two prospective, multicenter, controlled clinical trials. Ophthalmology, 113:992-1001.

Drolet DW, Nelson J, Tucker CE, et al. 2000. Pharmacokinetics and safety of an anti-vascular endothelial growth factor aptamer (NX1838) following injection into the vitreous humor of rhesus monkeys. Pharm Res, 17:1503-10.

Gragoudas ES, Adamis AP, Cunningham ET Jr, et al; VEGF Inhibition Study in Ocular Neovascularization (VISION) Clinical Trial Group. 2004. Pegaptanib for neovascular age-related macular degeneration. N Engl J Med, 351:2805-16.
Ishida S, Usui T, Yamashiro K, et al. 2003. VEGF164-mediated inflammation is required for pathological, but not physiological, ischemia-induced retinal neovascularization. J Exp Med, 198: 483-9.

Mennel S, Callizo J, Schmidt JC, et al. 2007. Acute retinal pigment epithelial tear in the untreated fellow eye following repeated bevacizumab (Avastin) injections. Acta Ophthalmol Scand, 85:689-90. Epub 2007 Apr 2.

Mitchell J, Bradley C. 2006. Quality of life in age-related macular degeneration: a review of the literature. Health Qual Life Outcomes, 21; 4:97.

Rosenfeld PJ, Brown DM, Heier JS, et al; MARINA Study Group. 2006a Ranibizumab for neovascular age-related macular degeneration. $N E n g l$ J Med, 355:1419-31.

Rosenfeld PJ, Rich RM, Lalwani GA. 2006b. Ranibizumab: Phase III clinical results. Ophthalmol Clin North Am, 19:361-72.

Rosenfeld PJ, Fung AE, Lalwani GA, et al. 2006c. Visual acuity outcomes following a variable-dosing regimen for ranibizumab (Lucentis) in neovascular AMD: the PrONTO Study. Abstract presented at the Association for Research in Vision and Ophthalmology, Fort Lauderdale, Florida, 30 April - 4 May 2006; Abstract 2958.

Spaide RF, Laud K, Fine HF, et al. 2006. Intravitreal bevacizumab treatment of choroidal neovascularization secondary to age-related macular degeneration. Retina, 26:383-90.

Macula Committee of the German Ophthalological Society (DOG) and the Professional Association of Ophthalmologists of Germany (BVA) 2006. Stellungnahme zu aktuellen therapeutischen Möglichkeiten bei der neovaskulären altersabhängigen Makuladegeneration (Comments on current therapeutic possibilities for neovascular age-related macular degeneration). Klin Monatsbl Augenheikd, 223:271-8.

Tilton RG, Chang KC, LeJeune WS, et al. 1999. Role for nitric oxide in the hyperpermeability and hemodynamic changes induced by intravenous VEGF. Invest Ophthalmol Vis Sci, 40:689-96.

Thakker GD, Hajjar DP, Muller WA, et al. 1999. The role of phospatidylinositol 3-kinase in vascular endothelial growth factor signaling. J Biol Chem, 274:10002-7.

Roche Products Limited. 2007. Summary of Product Characteristics: Avastin $25 \mathrm{mg} / \mathrm{ml}$ concentrate for solution for infusion. Last updated 12 November 2007. 
